\title{
Are immunosuppressant related to unfavorable outcomes in patients COVID-19 with autoimmune rheumatic disease?: A review of clinical evidence
}

\author{
Oki Nugraha Putra*, Hardiyono Hardiyono, Mia Arum Anggraini \\ Department of Clinical Pharmacy, Program Study of Pharmacy, Hang Tuah University, Arief Rahman Hakim 150, Surabaya, East Java-Indonesia
}

\begin{abstract}
The impact of immunosuppressant therapy in COVID-19 patients with autoimmune rheumatic disease remains unclear based on previous studies. Here, we reviewed the clinical evidence to evaluate COVID-19 patients with rheumatic disease outcomes, which previously used immunosuppressant therapy to control the disease. We used PubMed and Science Direct database to search literature up to April 2021 for publications with confirmed COVID-19 infection with rheumatic disease. The outcomes of this review were the infection rate of COVID-19 and the rate of hospitalization, ICU admission, and mortality. A total of 16 articles were included in this review. The overall rates of COVID-19 infection in patients with autoimmune rheumatic disease did not differ from the general population. Rheumatic disease patients who previously used hydroxychloroquine showed a similar infection risk of COVID-19 with those who did not use hydroxychloroquine. Furthermore, immunosuppressant therapies were associated with poor clinical outcomes, increase risk of hospitalization, ICU admission, and mortality, particularly in patients with comorbidities. The use of bDMARD, such as TNF- $\alpha$ inhibitor, showed a protective effect to reduce the risk of hospitalization and mortality. The administration of immunosuppressant therapy must be closely monitored in rheumatic disease patients due to unfavorable outcomes. More studies are urgently required to map risk factors of clinical outcomes with the specific immunosuppressant therapy and specific rheumatic disease.
\end{abstract}

Keywords:

COVID-19, Immunosuppressant, Autoimmune, Rheumatic disease, Outcomes

\section{INTRODUCTION}

Corona Virus-19 Disease (COVID-19) is caused by severe acute respiratory syndrome coronavirus-2 (SARS-CoV-2), including beta-coronavirus with an RNA core. The disease was first reported from Wuhan, China, in December 2019, and this infection has now been reported worldwide ${ }^{1,2}$. On 11 March 2020, WHO declared COVID-19 as a pandemic. In Indonesia, at the end of February 2021, it was estimated more than one million patients with COVID-19 were confirmed, with 35.014 cases of mortality ${ }^{3}$.

A high fatality rate was found in the elderly and comorbidities, especially in patients with chronic respiratory or cardiovascular disease, diabetes, hyper- tension, and cancer ${ }^{4}$. In addition, a high fatality rate has been reported in transplant patients or with autoimmune disease, especially in those receiving immunosuppressant for long treatment ${ }^{5}$. Since the pandemic, there has been concern about the risk of SARS-CoV-2 infection and complications in patients with autoimmune rheumatic disease ${ }^{6}$. Furthermore, these patients have a higher risk of infection because they are in a state of decreased immunity due to immunosuppressant treatment ${ }^{7}$. In addition, immunosuppressant therapy suppresses the abnormal immune response in COVID-19, which is responsible for severe complications, such as interstitial pneumonia ${ }^{8}$. Additionally, exposure of glucocorticoid and increase of comorbidities among patients with an autoimmune rheumatic disease (obesity, chronic lung disease, diabetes,

*Corresponding author:

*oki Nugraha Putra oki.nugraha@hangtuah.ac.id 
cardiovascular disease) may impact on increasing the high risk of a worse prognosis .

Immunosuppressant agents, such as corticosteroids, conventional synthetic disease-modifying anti-rheumatic drugs (csDMARDs), biologic DMARD (bDMARDs), and targeted synthetic DMARD(tsDMARDs), are commonly prescribed to treat numerous autoimmune rheumatic disease, such as rheumatoid arthritis (RA), systemic lupus erythematosus (SLE), etc. as well as for prophylaxis after organ transplantation procedures ${ }^{10}$. The impact of COVID-19 on patients with rheumatic diseases is currently unknown. It is unknown whether patients with rheumatic disease have a worse outcome because of their immunocompromised status or whether they have a better outcome because of anti-rheumatic therapies that reduce hyper-inflammation. In version 2 of the American College of Rheumatology guidance for the management of rheumatic disease in adult patients during the COVID-19 pandemic stated that no specific rheumatic diseases were identified as risk factors for poor outcomes COVID-19. During the pandemic, some studies reported different results regarding clinical outcomes in patients with autoimmune rheumatic disease and COVID-19.

Some studies reported that rheumatic disease was not associated with the risk of SARS-CoV-2 infection and poor clinical outcomes, while other studies are not. However, most of the studies were case reports or case series and survey-based designs. Whether poor clinical outcomes were because of underlying autoimmune disease or immunosuppressant medicines were unclear in previously published studies. Most of the studies did not eliminate the confounding variables such as age, sex, and comorbidities on the clinical outcomes in patients using immunosuppressant. A small number of patients, wide variations of methodology, and lack of generalization to the population were limitations of most studies.

Therefore, we conducted a review to summarize the impact of immunosuppressant therapy in patients with autoimmune rheumatic disease and COVID-19 from the available evidence that described the clinical characteristics and outcomes of COVID-19 patients with autoimmune rheumatic disease.

\section{METHOD}

This review was performed based on the Preferred Reporting Items for Systematic Reviews and Meta-Analyses (PRISMA) guidelines.

\subsection{Search strategy}

We used PubMed and Science Direct database to comprehensively search articles published up to April 2021 between March and April 2021. We used keywords "autoimmune disease", "COVID-19", "rheumatic disease", "rheumatoid arthritis", "systemic lupus erythematosus", "immunosuppressant", and "immunosuppressive" to search the article in the database. Boolean operator with "OR" and "AND" was used to search articles more specific.

\subsection{Study selection and data extraction}

All studies involving COVID-19 infected rheumatic patients epidemiological and clinical information were listed. The inclusion criteria in this review were articles focusing on using immunosuppressant therapy to the prevalence of SARS-CoV-2 and clinical outcomes (hospitalization, ICU admission, and mortality) in COVID-19 patients with autoimmune rheumatic disease. Multivariate analysis or logistic regression must be done in the included articles to associate immunosuppressants with clinical outcomes. Studies with a cohort, cross-sectional, and case-control were enrolled in this review. We excluded studies in commentary, review, letter to the editor, case report, and case series. The following information, such as name of authors, study design, sample size, age, sex, type of autoimmune rheumatic diseases, and outcomes, was extracted and summarized in the table.

\subsection{Outcomes}

The primary outcomes of the included studies were infection rate of SARS-CoV-2, risk of hospitalization, ICU admission, the use of a ventilator, and mortality among patients with rheumatic disease.

\section{RESULTS}

A total of 16 studies met the inclusion criteria and were included in this present review involving 42.130 patients with autoimmune rheumatic disease. The PRISMA flowchart for article inclusion is shown in Figure 1. The summary characteristic of the included studies is summarized in Table 1. All of the included studies were observational study, either prospective or retrospective.

\section{DISCUSSION}

This present review has summarized and evaluated the available published literature describing unfavorable outcomes of immunosuppressant therapy in patients with rheumatic disease to the infection risk of COVID-19 and worsen clinical outcomes. Patients with autoimmune rheumatic disease require special attention during the COVID-19 pandemic. They are classified as high risk for infections and other unfavorable effects resulting from their immunocompromised state. About $71 \%$ of the patients were female, corresponding to the 


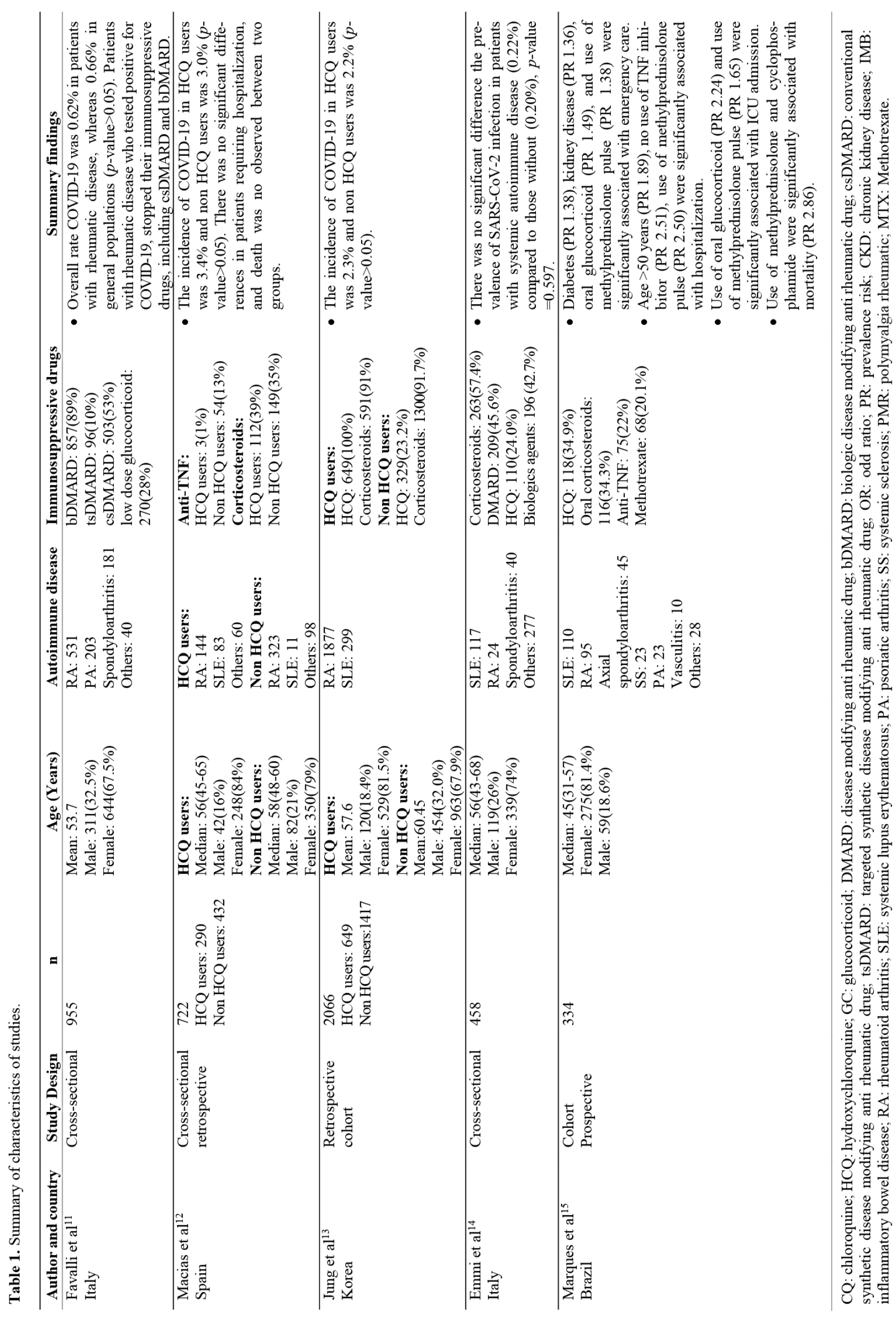




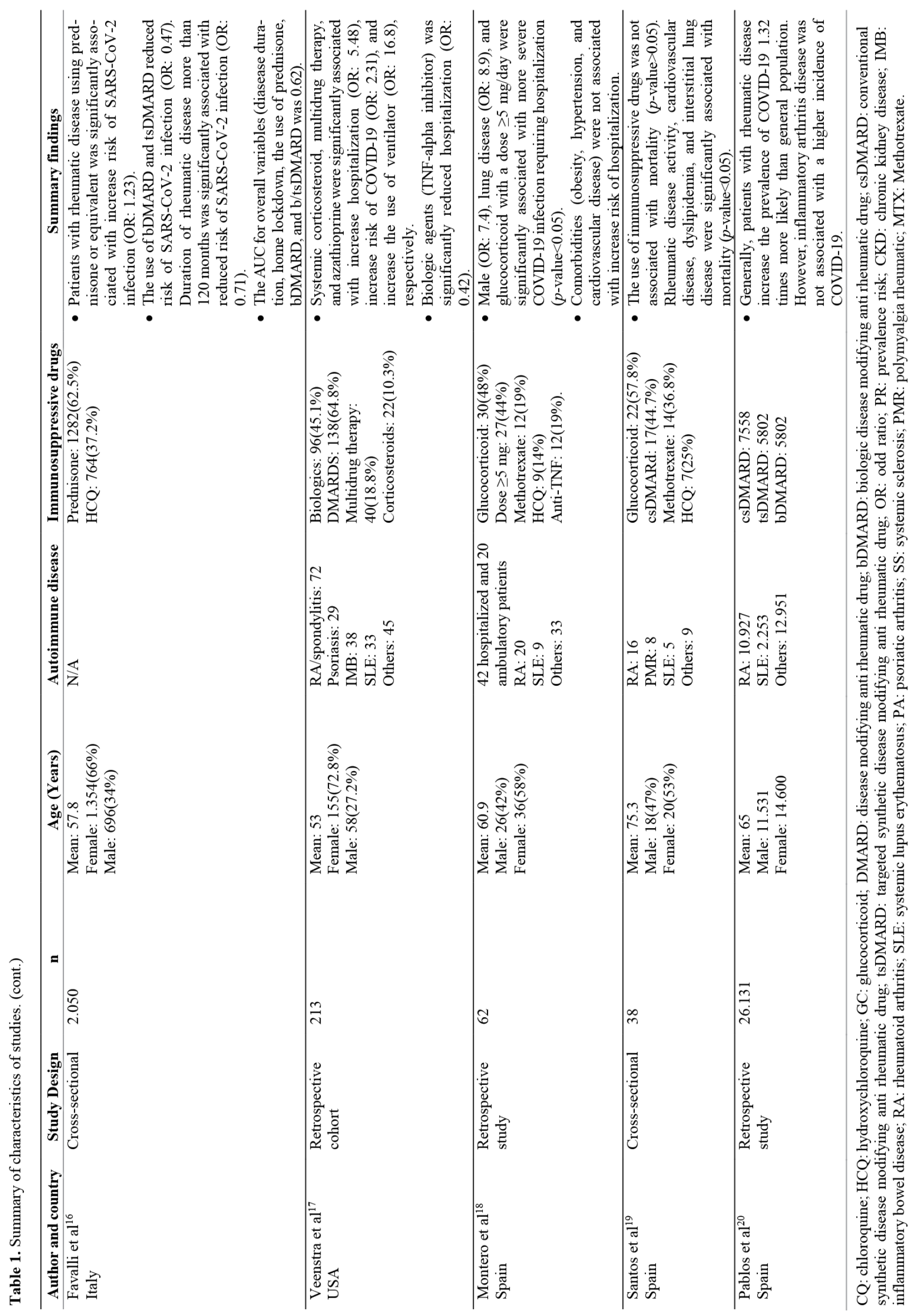




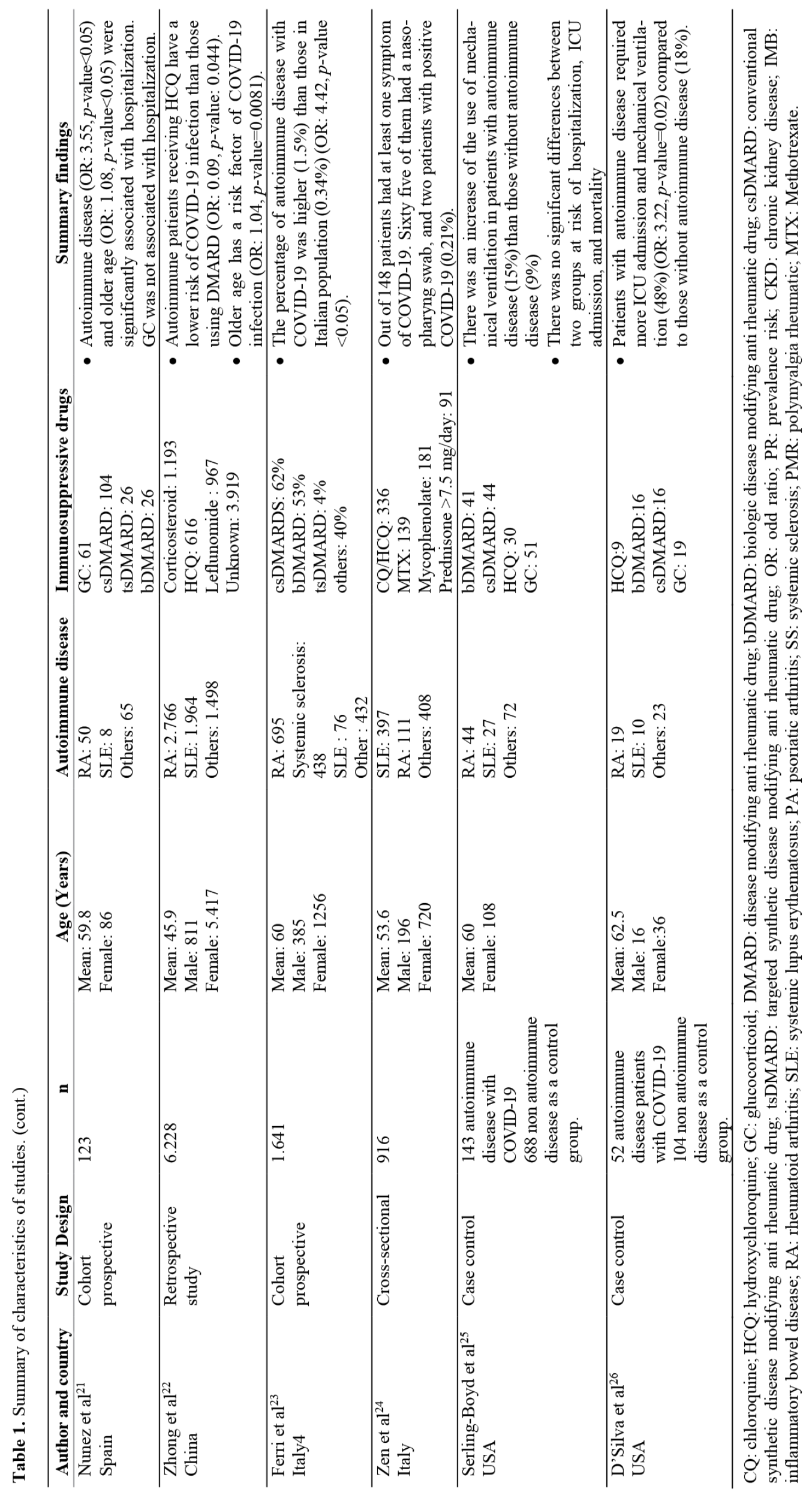




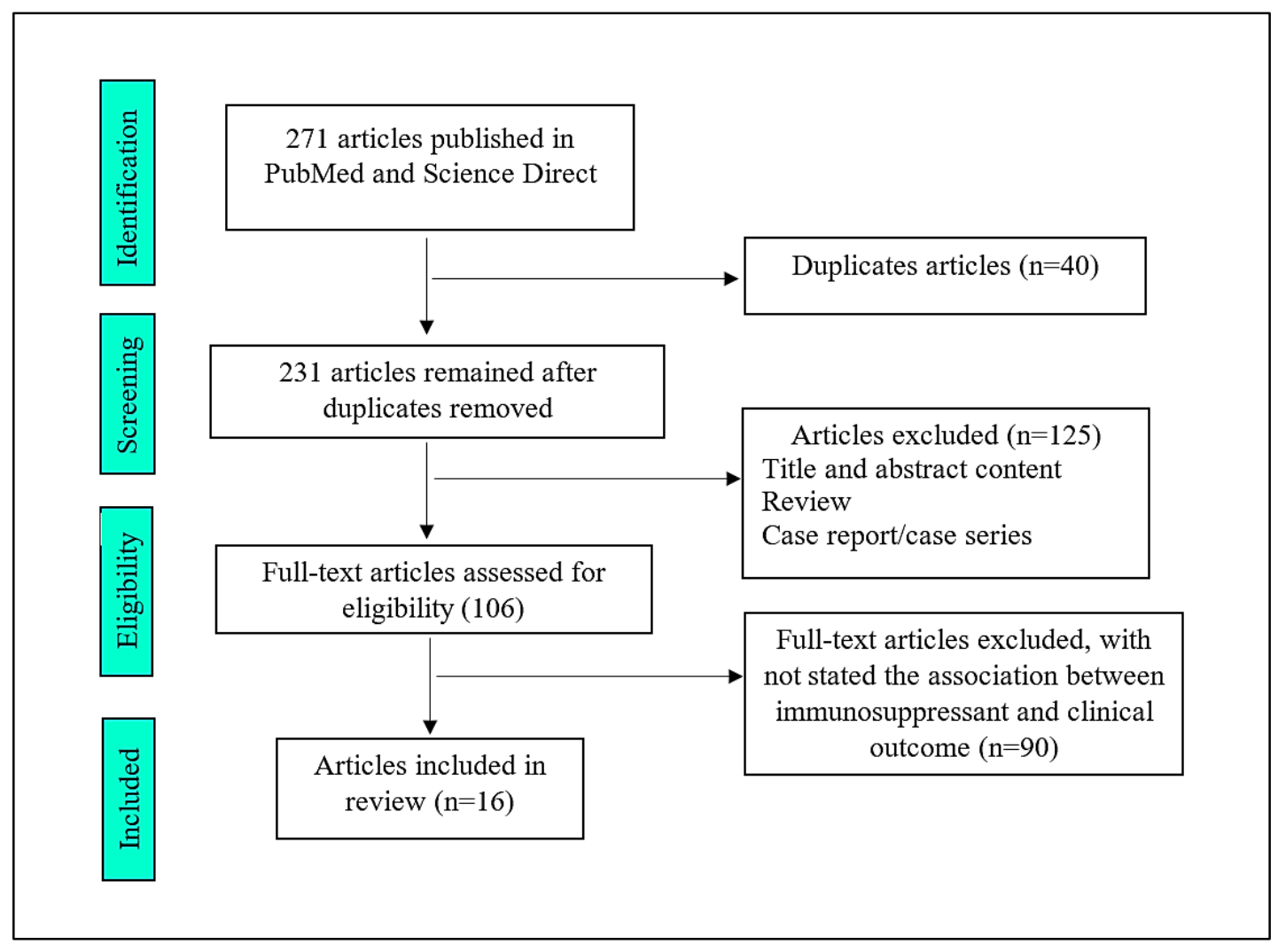

Figure 1. PRISMA flowchart.

natural history of female predominance in autoimmune diseases. Compared to the meta-analysis study by $\mathrm{Xu}$ et al, which reported that $64 \%$ of patients were female. Because that study involved 31 articles with 1138 patients, a lower number of patients compared to our review ${ }^{27}$. However, our study was consistent with the Global Registry, stating about $76 \%$ of patients were female ${ }^{28}$. The number of patients with rheumatoid arthritis in Indonesia is currently estimated at not less than 1.3 million, with calculations based on the prevalence of AR in the world between $0.5-1 \%{ }^{29}$. The outcomes of each study were identified as accumulated outcomes without further differentiation by rheumatic disease type ${ }^{30}$.

Most studies used hydroxychloroquine as a part of rheumatic disease treatment. The possible antiviral effects of chloroquine and hydroxychloroquine in the pandemic's early phase were interesting aspects that seemed to prevent SARS-CoV-2 endocytosis in alveolar epithelial cells in some in vitro studies. These drugs were given Emergency Authorization Used (EAU) by FDA to treat COVID-19 in April 2020. However, in June 2020, both drugs were suspended for COVID-19 management because of harmful effects, QT prolongation in several studies ${ }^{30-32}$ including in Indonesia. National Agency of Drug and Food Control of Indonesia announced that hydroxychloroquine was permitted to treat autoimmune rheumatic disease, while chloroquine was not recommended due to harmful toxicity ${ }^{33}$. Due to its antiviral mechanism, hydroxychloroquine has been proposed to prevent SARS-CoV infection. However, this hypothesis was unclear whether the prophylactic effect of hydroxychloroquine could be observed in healthy individuals or patients with a specific disease. In the present review, we found eight studies that reported the overall rate of SARS-CoV-2 infection among autoimmune rheumatic disease ranged from $0.22 \%$ to $3.0 \%$, and it was no different with the general population without rheumatic disease. This review was similar to a study in Indonesia, which reported that among 570 autoimmune rheumatic diseases, eleven patients were confirmed SARS-CoV-2 or approximately $1.92 \%{ }^{34}$.

Furthermore, there was no significant difference in the prevalence of SARS-CoV-2 infection between HCQ users and non HCQ users among patients with rheumatic disease in two studies by Macias et $\mathrm{al}^{12}$. (3.4\% vs. $3.0 \%$ ) and Jung et $\mathrm{al}^{13}$. (2.3\% vs. $\left.2.2 \%\right)$. A lower rate of SARS-CoV-2 infection ( $0.43 \% ; 27 / 6228)$ among patients with rheumatic disease was reported by Zhong et al. in Hubei province, China. In that study, hydroxychloroquine was significantly associated with reduced risk of COVID-19 compared to those taking other DMARDs (OR 0.09, CI 95\% 0.21-5.40, $p$-value $<0,05)^{22}$. However, in a study by Zhong et $\mathrm{al}^{22}$., the percentage of patients who were taking hydroxychloroquine was lower (26.7\%) compared to the study by Macias et $\mathrm{al}^{12}$. $(40.16 \%)$ and Jung et $\mathrm{al}^{13}$. (31.4\%), respectively. Both studies reported COVID-19 incidence and severity were not significantly different in patients with autoimmune rheumatic disease 
treated with or without hydroxychloroquine ${ }^{12,13}$.

The study by Machias et $\mathrm{al}^{12}$., stated that the overall incidence of COVID-19 was $0.164 \%$ (95\% CI= $0.16 \%-0.17 \%$ ) cases. This figure is within the $95 \% \mathrm{CI}$ of confirmed COVID-19 found either in patients with or without taking HCQ. This shows that incidence of confirmed SARS-CoV-2 infection among patients with autoimmune rheumatic disease, with or without HCQ, is similar to the incidence of SARS-CoV-2 infection in general population. There was no clinical efficacy associated with the prophylaxis of CQ and HCQ in randomized controlled trials. The efficacy of $\mathrm{CQ} / \mathrm{HCQ}$ for post-exposure prophylaxis has rarely been studied in randomized control trials with long-term supervision of patients and their contacts. The drug's toxicity, in particular cardiac toxicity, has often outweighed its benefits, in contrast to the treatment of malaria. Patients with COVID-19 who were administered CQ/HCQ did not show any clinical benefit in a recent meta-analysis of 12 studies $^{35}$. Furthermore, both of the two largest randomized controlled trials (RECOVERY ${ }^{36}$ and WHO SOLIDARITY ${ }^{37}$ ) confirmed a lack of clinical benefit of chloroquine and hydroxychloroquine for COVID-19 patients.

Another therapy for autoimmune rheumatic disease, especially in SLE, is a glucocorticoid that can be used with other DMARDs based on the severity of the disease. Rheumatic disease patients administered corticosteroids, especially in high doses, are at risk for severe infection ${ }^{38}$. Glucocorticoids were identified as a significant risk factor for bacterial infection in a cohort study of over 15,000 patients with RA over the age of 65. Glucocorticoid doubled the rate of serious bacterial infections compared to methotrexate use, with a clear dose-response relationship for dosages greater than 5 $\mathrm{mg} /$ day and $20 \mathrm{mg} /$ day $^{39}$.

Glucocorticoids have been used to manage COVID-19 patients, particularly those with cytokine storm and acute respiratory distress syndrome (ARDS). Several studies have reported slower virus clearance, which may worsen the patient's condition. The Indonesian Rheumatology Association recommends a dose of prednisone as initial therapy of $20 \mathrm{mg} /$ week for mild SLE and $0.5 \mathrm{mg} / \mathrm{kg} /$ day for moderate to severe SLE. In the maintenance phase, the recommended dose of prednisone is $7.5 \mathrm{mg} /$ day for all degrees of SLE in combination with other immunosuppressant therapy ${ }^{40}$.

This review found four articles discussing the unfavorable effects of corticosteroids in autoimmune rheumatic disease, including increased risk of SARSCoV-2 infection, hospitalization, and mortality. A study by Favalli et al, reported that prednisone at a dose $>2.5$ $\mathrm{mg} /$ day was significantly associated with the development of SARS-CoV-2 infection ${ }^{16}$. However, a metaanalysis study indicated that prednisone or equivalent for the short term has no impact or little in infection risk. Interestingly, an observational study evaluating a different dose of glucocorticoid (low, medium, and high dose) demonstrated that patients receiving a medium to high dose ( $\geq 7.5 \mathrm{mg} /$ day) of prednisolone or equivalent were associated with increased risk of infection and severe infection ${ }^{41}$. However, the odds may differ for each disease treated with glucocorticoid. A study by Fardet et al. reported that the adjusted hazard ratio of infection was varied in patients receiving glucocorticoid. The hazard ratio was 2.01 (CI 95\%=1.83-2.19) for cutaneous cellulitis, and 5.84 (CI 95\%=5.61-6.08) for lower respiratory tract infection ${ }^{42}$.

We found the use of methylprednisolone pulse was significantly associated with emergency care, hospitalization, ICU admission, and mortality, reported by Marques and co-workers ${ }^{15}$. Intravenous methylprednisolone pulse was administered with a dose of $0.5-1$ $\mathrm{g} /$ day for three consecutive days. It is well assumed that because of their higher potency and the onset of action is faster than oral prednisone, the administration of methylprednisolone pulses is indicated for severe SLE when a rapid effect is crucial. This therapy aims to avoid high-dose and long-term oral prednisone, induce a faster tapering, and reduce the cumulative dose of corticesteroid and adverse effects ${ }^{43}$. A higher infection rate was observed in patients with SLE receiving methylprednisolone with the dose $>1500 \mathrm{mg}$ those less than 1500 $\mathrm{mg}$. The infection rate was $9.1 \%$ in those who receive methylprednisolone with the dose $>1500 \mathrm{mg}$. Meanwhile, no patients suffered an infection in a $<1500 \mathrm{mg}^{44}$. In contrast, the study by Nuñez et al. stated that glucocorticoids were not associated with the risk of hospitalization. However, the study did not mention the dose and duration of glucocorticoid use and the relatively small sample size of 123 patients. In addition, only 54 of the 123 patients underwent treatment at the hospital. From the multivariate analysis results, there was no relationship between the use of glucocorticoids and the risk of hospitalization $^{21}$.

Infections represent one of the adverse effects of high-dose corticosteroids, causing an increased risk of morbidity and mortality in patients with SLE. The factors precipitating infection in patients with SLE are disease activity and the administration of immunosuppressant agents, particularly glucocorticoid ${ }^{45}$. Glucocorticoid suppresses cell-mediated immunity by inhibiting IL-1, IL-2, IL-6, dan IFN-gamma. The inhibition of IL-2 reduces $\mathrm{T}$ lymphocyte proliferation and inhibits the clonal development expansion of B lymphocytes responsible for producing immunoglobulin. Furthermore, glucocorticoids weaken macrophage activity and impair phagocytosis activity ${ }^{46}$. The Indonesia Rheumatology Association recommended corticosteroids can be given to patients with confirmed COVID-19 who are asymptomatic or with mild-moderate symptoms of infection with the smallest effective dose according to their rheu- 
matic disease activity. In COVID-19 patients with signs of severe infection, the determination of the corticosteroid dosage takes into account the clinical conditions and weights risk-benefit ratio for each patient ${ }^{47}$.

Surprisingly, five studies reported that the use of bDMARD, particularly TNF- $\alpha$ inhibitor, was significantly associated with a low risk of SARS-CoV-2 infection and hospitalization. TNF- $\alpha$ is a pro-inflammatory cytokine produced by activated monocytes. It involved almost in acute inflammatory reactions, acting as an inducer of inflammation. COVID-19 patients were proposed to have high inflammatory biomarkers, such as IL-6, IL-8, and TNF- $\alpha$. A blockaded high level of TNF- $\alpha$ has beneficial effects by improving prognosis and reducing organ damage in inflammatory diseases, such as rheumatic disease and COVID-1948. A recent study by Del Valle and co-workers indicated that in COVID-19 patients, TNF- $\alpha$ level was high on the first admission. It was a strong and independent predictor of a patient's survival ( $p$-value $<0.05$ ). The level of TNF- $\alpha$ gradually decreased after the administration of tocilizumab, an IL-6 inhibitor ${ }^{49}$. Several clinical trials demonstrated that the use of TNF- $\alpha$ inhibitors, including etarnecept, infliximab, and adalimumab, relieves inflammation of the joint, reduces severe joint progression, and improves physical function in patients with advanced rheumatoid arthritis $^{50-52}$.

Furthermore, the administration of TNF- $\alpha$ inhibitor in patients with rheumatoid arthritis is more efficacious to promote better symptoms and clinical signs than methotrexate alone. However, The Indonesia Rheumatology Association recommended that the bDMARD administered for rheumatic disease patients with COVID-19 is an IL-6 inhibitor after COVID-19 infection is over. TNF- $\alpha$ inhibitors should be avoided based on this guideline ${ }^{53}$.

Several studies in the present review reported comorbidities, including cardiovascular disease, diabetes, chronic respiratory disease, as significant risk factors for hospitalization. Some classical cardiovascular risk factors such as smoking, insulin resistance, dyslipidemia, arterial hypertension, and low baseline of vitamin D influenced cardiovascular comorbidities in patients with rheumatic disease ${ }^{52-54}$. Meanwhile, methotrexate and TNF- $\alpha$ inhibitor were protective factors ${ }^{55,56}$. The development of cardiovascular events among rheumatic disease patients was observed in 10 years and doubled within 12 months ${ }^{53}$. Metabolic syndrome and hypertension were commonly found in patients with rheumatoid arthritis and SLE, with an estimated $14-38 \%$ prevalence for metabolic syndrome and $23-66 \%$ for hypertension ${ }^{57,58}$. Some studies reported cardiovascular disease (RR: 2.25$)^{59}$, hypertension (RR: 2.39$)^{60}$, and respiratory disease (OR: $5.15)^{61}$ was associated with mortality on COVID-19 patients with rheumatic disease.

Meanwhile, the study by Montero et al. showed the comorbidities were not associated with hospitalization. It was because of the limited number of patients and the study design was a retrospective study $^{18}$. An effort should be taken to prevent mortality in patients with rheumatic disease. Angiotensin receptor blockers (ARBs) and angiotensin-converting enzyme inhibitors (ACEi) were clinically proven to avoid severity and mortality in COVID-19 patients, particularly with comorbidities $^{62}$.

There was some limitation of our review. First, all of the studies did not report the dose and duration of the immunosuppressant therapy to treat rheumatic disease. These aspects will impact the severity and prognosis of patients. Second, some studies have a limited number of patients and with crosssectional design. Therefore, the results did not reflect the generalized populations directly. Third, the details of the outcomes were unavailable for specific rheumatic disease and specific immunosuppressant therapy. The immunomodulatory effects among immunosuppressant therapy such as csDMARD, bDMARD, tsDMARD may be different.

\section{CONCLUSION}

The use of immunosuppressant therapy in rheumatic disease patients before the onset of COVID19 may have unfavorable effects with poor prognosis outcomes. Overall, corticosteroid use, particularly in the moderate to high dose, was associated with poor clinical outcomes. TNF- $\alpha$ inhibitors may be helpful to reduce harmful prognostic factors. Comorbidities in rheumatic disease patients should be closely supervised to minimize the risk for morbidity and mortality. Rheumatologists, pharmacists, and other healthcare teams should collaborate to monitor the prognosis and report the outcomes for a specific rheumatic disease and specific immunosuppressant therapy. This study emphasized further study with larger sample size, multicenter, more detailed treatment, and disease-specific outcomes.

\section{Conflict of interest}

All authors declare no conflict of interest in this review

\section{Funding}

All authors declare no supporting funding from any agency in this review

\section{Ethics approval}

None to declare.

Article info:

Received May 12, 2021

Received in revised form August 9, 2021

Accepted October 28, 2021 


\section{REFERENCES}

1. Huang C, Wang Y, Li X, Ren L, Zhao J, Hu Y, et al. Clinical features of patients infected with 2019 novel coronavirus in Wuhan, China. Lancet. 2020;395:497-506.

2. Lu R, Zhao X, Li J, Niu P, Yang B, Wu H, et al. Genomic characterization and epidemiology of 2019 novel coronavirus: Implications for virus origin and receptor binding. Lancet. 2020; 395:565-74.

3. Task force of COVID-19 of Indonesia. Available from: https:// covid19.go.id.

4. Onder G, Rezza G, Brusaferro S. Case-fatality rate and characteristics of patients dying in relation to COVID-19 in Italy. JAMA. 2020;323(8):1775-76.

5. Figueroa-Parra G, Aguirre-Garcia GM, Gamboa-Alonso CM, Camacho-Ortiz A, Galarza-Delgado DA. Are my patients with rheumatic diseases at higher risk of COVID-19?. Ann Rheum Dis. 2020;79(6):839-40.

6. Favalli EG, Ingegnoli F, De Lucia O, Cincinelli G, Cimaz R, Caporali R. COVID-19 infection and rheumatoid arthritis: Faraway, so close!. Autoimmun Rev. 2020;19(5):102523.

7. Meroni PL, Zavaglia D, Girmenia C. Vaccinations in adults with rheumatoid arthritis in an era of new disease-modifying antirheumatic drugs. Clin Exp Rheumatol. 2018;36:317-28.

8. Mehta P, McAuley DF, Brown M, Sanchez E, Tattersall RS, Manson JJ. COVID-19: consider cytokine storm syndromes and immunosuppression. Lancet. 2020;395:1033-34.

9. Hasseli R, Mueller-Ladner U, Hoyer BF, Krause A, Lorenz H, Pfeil A, et al. Older age, comorbidity, glucocorticoid use and disease activity are risk factors for COVID-19 hospitalization in patients with inflammatory rheumatic and musculoskeletal diseases. RMD Open. 2021;7(1):e001464.

10. Caporali R, Caprioli M, Bobbio-Pallavicini F. DMARDs and infections in rheumatoid arthritis. Autoimmun Rev. 2008;8(2): 139-43.

11. Favalli EG, Monti S, Ingegnoli F, Balduzzi S, Caporali R, Montecucco C. Incidence of COVID-19 in patients with rheumatic diseases treated with targeted immunosuppressive drugs: what can we learn from observational data?. Arthritis Rheumatol. 2020;72(10):1600-06.

12. Macias J, Gonzalez-Moreno P, Sanchez-Garcia E, MorilloVerdugo R, Perez-Venegas JJ, Pinilla A, et al. Similar incidence of coronavirus disease 2019 (COVID-19) in patients with rheumatic disease with and without hydroxychloroquine therapy. PLoS One. 2021;16(4):e0249036.

13. Jung SY, Kim MS, Kim MC, Choi SH, Chung JW, Chou ST. Effect of hydroxychloroquine pre-exposure on infection with SARS-CoV-2 in rheumatic disease patients: a population-based cohort study. Clin Microbiol Infect. 2021;27:611-7.

14. Emmi G, Bettiol A, Mattioli I, Silvestri E, Di Scala G, Urban ML, et al. SARS-CoV-2 infection among patients with systemic autoimmune disease. Autoimmun Rev. 2020;19(7):102575.

15. Marques CDL, Kakehasi AM, Pinheiro MM, Mota LMH, Albuquerque CP, Silva CR, et al. High levels of immunosuppression are related to unfavourable outcomes in hospitalized patients with rheumatic diseases and COVID-19: first results of ReumaCov Brazil registry. RMD Open. 2021;7:e001461.

16. Favalli EG, Bugatti S, Klersy C, Biggioggero M, Rossi S, De Lucia $\mathrm{O}$, et al. Impact of corticosteroids and immunosuppressive therapies on symptomatic SARS-CoV-2 infection in a large cohort of patients with chronic inflammatory arthritis. Arthritis Res Ther. 2020;22:290.

17. Veenstra J, Buechler CR, Robinson G, Chapman S, Adelman M, Tisack A, et al. Antecedent immunosuppressive therapy for immune-mediated inflammatory diseases in the setting of a COVID-19 outbreak. J Am Acad Dermatol. 2020;83(6):1696-703.

18. Montero F, Martinez-Barrio J, Serrano-Benavente B, Gonzalez T, Rivera J, Collada JM, et al. Coronavirus disease 2019 (COVID-19) in autoimmune and inflammatory conditions: clinical characteristics of poor outcomes. Rheumatol Int. 2020; 40:1593-98.

19. Santos CS, Morales CM, Alvarez ED, Castro CA, Robles AL, Sandoval TP. Determinants of COVID-19 disease severity in patients with underlying rheumatic disease. Clin Rheumatol. 2020;39:2789-96.

20. Pablos JL, Abasolo L, Alvaro-Gracia JM, Blanco FJ, Blanco R, Castrejón I, et al. Prevalence of hospital PCR-confirmed COVID19 cases in patients with chronic inflammatory and autoimmune rheumatic diseases. Ann Rheum Dis. 2020;79(9):1170-73.

21. Nuñez DDF, Leon L, Mucientes A, Rodriguez-Rodriguez L, Font Urgelles J, Madrid García A, et al. Risk factors for hospital admissions related to COVID-19 in patients with autoimmune inflammatory rheumatic diseases. Ann Rheum Dis. 2020;79(11): 1393-99.

22. Zhong J, Shen G, Yang H, Huang A, Chen X, Dong L, et al. COVID-19 in patients with rheumatic disease in Hubei province, China: a multicentre retrospective observational study. Lancet Rheumatol. 2020;2(9):E557-64.

23. Ferri C, Giuggioli D, Raimondo V, L'Andolina M, Tavoni A, Cecchetti R, et al. COVID-19 and rheumatic autoimmune systemic diseases: report of a large Italian patients series. Clin Rheumatol. 2020;39(11):3195-204.

24. Zen M, Fuzzi E, Astorri D, Saccon F, Padoan R, Ienna L, et al. SARS-CoV-2 infection in patients with autoimmune rheumatic diseases in northeast Italy: A cross-sectional study on 916 patients. J Autoimmun. 2020;112:102502.

25. Serling-Boyd N, D'Silva KM, Hsu TYT, Wallwork R, Fu X, Gravallese EM, et al. Coronavirus disease 2019 outcomes among patients with rheumatic diseases 6 months into the pandemic. Ann Rheum Dis. 2020;80(5):660-6.

26. D'Silva KM, Serling-Boyd N, Wallwork R, Hsu T, Fu X, Gravallese EM, et al. Clinical characteristics and outcomes of patients with coronavirus disease 2019 (COVID-19) and rheumatic disease: A comparative cohort study from a US 'hot spot'. Ann Rheum Dis. 2020;79(9);1156-62.

27. Xu C, Yi Z, Cai R, Chen R, Yu-Hor TB, Mu R. Clinical outcomes of COVID-19 in patients with rheumatic disease: a systematic review and meta-analysis of global data. Autoimmun Rev. 2021;20(4):102778.

28. Data from the COVID-19 Global Rheumatology Alliance Global Registry. Available from: https://rheum-covid.org/updates/combined-data.html.

29. Recommendation of the Indonesian Rheumatologist Association. Diagnosis and management of Rheumatoid Arthritis. Jakarta: Indonesian Rheumatology Association; 2021.

30. Ehrenfeld M, Tincani A, Andreoli L, Cattalini M, Greenbaum A, Kanduc D, et al. COVID-19 and autoimmunity. Autoimmun Rev. 2020;19(8):102597.

31. Becker ML, Snijders D, van Gemeren CW, Kingma HJ, van Lelyveld SFL, Giezen TJ. QTc prolongation in COVID-19 patients using chloroquine. Cardiovasc Toxicol. 2021;21:314-21.

32. Shah RR. Chloroquine and hydroxychloroquine for COVID-19: perspectives on their failure for repurposing. J Clin Pharm Ther. 2021;46:17-27.

33. National Agency of Drug and Food Control of Indonesia. A clarification about the withdrawn of emergency use authorization hydroxychloroquine and chloroquine to COVID-19 [document on the Internet]. Available from: https://www.pom.go.id/new/ view/more/klarifikasi/121/PENJELASAN-BADAN-POM-RI-TENTANG-Pencabutan-Emergency-Use-Authorization Hidroksiklorokuin-dan-Klorokuin-untuk-Pengobatan-COVID-19.html.

34. Hidayat R, Isbagio H, Ariane A, Parlindungan F, Hamijoyo L, Suarjana IY. Characteristics of patients with autoimmune rheumatic disease in the era of COVID-19 pandemic in Indonesia. IJR. 2020;12(1):251-8.

35. Ullah W, Abdullah HM, Roomi S, Sattar Y, Almas T, Gowda $\mathrm{SN}$, et al. Safety and efficacy of hydroxychloroquine in COVID- 
19: a systematic review and meta-analysis. J Clin Med Res. 2020; 12(8):483-91.

36. Horby P, Mafham M, Linsell L, Bell JL, Staplin N, Emberson JR, et al. Effect of hydroxychloroquine in hospitalized patients with Covid-19. N Engl J Med. 2020;383(21):2030-40.

37. Pan H, Peto R, Karim QA, Alejandria M, Henao-Restrepo AM, García CH, et al. Repurposed antiviral drugs for COVID-19; interim WHO solidarity trial results. N Engl J Med. 2021;384: 497-511.

38. Waki D, Nishimura K, Tokumasu H, Kadoba K, Mukoyama H, Saito $\mathrm{R}$, et al. Initial high-dose corti-costeroids and renal impairment are risk factors for early severe infections in elderly patients with antineutrophil cytoplasmic autoantibody-associated vasculitis: A retrospective observational study. Medicine. 2020; 99(8):e19173.

39. Schneeweiss S, Setoguchi S, Weinblatt ME, Setoguchi S, Katz JN, Avorn J, et al. Anti-tumor necrosis factor alpha therapy and the risk of serious bacterial infections in elderly patients with rheumatoid arthritis. Arthritis Rheum. 2007;56(6):1754-64.

40. Recommendation of the Indonesian Rheumatologist Association. Diagnosis and management of systemic lupus erythematosus. Jakarta: Indonesian Rheumatology Association; 2019.

41. Youssef J, Novosad SA, Winthrop KL. Infection risk and safety of corticosteroid use. Rheum Dis Clin N Am. 2015;42(1):157-76.

42. Fardet L, Petersen I, Nazareth I. Common infections in patients prescribed systemic glucocorticoids in primary care: a populationbased cohort study. PLoS Med. 2016;13(5):e1002024.

43. Fanouriakis A, Kostopoulou M, Alunno A, Aringer M, Bajema I, Boletis JN, et al. 2019 update of the EULAR recommendations for the management of systemic lupus erythematosus. Ann Rheum Dis. 2019;78:736-45.

44. Danza A, Borgia I, Narvaez JI, Baccelli A, Amigo C, Rebella M, et al. Intravenous pulses of methylprednisolone to treat flares of immune-mediated diseases: how much, how long?. Lupus. 2018; 27(7):1177-84.

45. Danza A, Ruiz-Irastorza G. Infection risk in systemic lupus erythematosus patients: susceptibility factors and preventive strategies. Lupus. 2013;22(12):1286-94.

46. Strehl C, Ehlers L, Gaber T, Buttgereit F. Glucocorticoid - allrounders tackling the versatile players of the immune system. Front Immunol. 2019;10:1744.

47. Recommendation of the Indonesian Rheumatologist Association. Management of autoimmune in the pandemic COVID-19 era. Jakarta: Indonesian Rheumatology Association; 2020.

48. Chen XY, Yan BX, Man XY. TNF- $\alpha$ inhibitor may be effective for severe COVID-10: learning from toxic epidermal necrolysis. Ther Adv Respir Dis. 2020;14:1-7.

49. Dal Valle DM, Kim-Schulze S, Huang HH, Beckmann ND, Nirenberg S, Wang B, et al. An inflammatory cytokine signature predicts COVID-19 severity and survival. Nat Med. 2020;26(10): $1636-43$.

50. Kekow J, Moots RJ, Emery P, Durez P, Koenig A, Singh A, et al. Patients-reported outcomes improve with etarnecept plus methotrexate in active early rheumatoid arthritis and the improvement is strongly associated with remission: The COMET trial. Ann Rheum Dis. 2010;69(1):222-5.

51. Harouni B, Cividino A, Stewart J, Guerette B, Keystone EC. Safety and effectiveness of adalimumab in a clinical setting that reflects Canadian standard of care for the treatment of rheumatoid arthritis (RA): results from the CanACT study. BMC Musculoskelet Disord. 2011;12:261.

52. Murphy D, Mattey D, Hutchinson D. Anti-citrullinated protein antibody positive rheumatoid arthritis is primarily determined by rheumatoid factor titre and the shared epitope rather than smoking per se. PLoS One. 2017;12(7):e0180655.

53. Ruscitti P, Ursini F, Cipriani P, Liakouli V, Carubbi F, Berardicurti $\mathrm{O}$, et al. Poor clinical responses in rheumatoid arthritis is the main risk factor for diabetes development in the short-term: a 1-year, single-centre, longitudinal study. PLoS One. 2017;12(7):e0181203.

54. Radner H, Lesperance T, Accort NA, Solomon DH. Incidence and prevalence of cardiovascular risk factors among patients with rheumatoid arthritis, psoriasis, or psoriatic arthritis. Arthritis Care Res (Hoboken). 2017;69(10):1510-18.

55. Roubille C, Richer V, Starnino T, McCourt C, McFarlane A, Fleming $\mathrm{P}$, et al. The effects of tumor necrosis factor inhibitors, methotrexate, non-steroidal anti-inflammatory drugs and corticosteroids on cardiovascular events in rheumatoid arthritis, psoriasis and psoriatic arthritis: a systematic review and metaanalysis. Ann Rheum Dis. 2015;74(3):480-9.

56. Mangoni AA, Baghadi LR, Shanahan EM, Wiese MD, Tomassi $\mathrm{S}$, Elliot D, et al. Methotrexate, blood pressure and markers of arterial function in patients with rheumatoid arthritis: a repeated cross-sectional study. Ther Adv Musculoskelet Dis. 2017;9(9): 213-29.

57. Kerekes G, Nurmohamed MT, González-Gay MA, Seres I, Paragh G, Kardos Z, et al. Rheumatoid arthritis and metabolic syndrome. Nat Rev Rheumatol. 2014;10(11):691-6.

58. Munguia-Realpozo P, Mendoza-Pinto C, Sierra Benito C, Escarcega RO, Garcia-Carrasco M, Martinez SM, et al. Systemic lupus erythematosus and hypertension. Autoimmun Rev. 2019;18(10): 102371.

59. Pranata R, Huang I, Lim MA, Wahjoepramono EJ, July J. Impact of cerebrovascular and cardiovascular diseases on mortality and severity of COVID-19-systematic review, meta-analysis, and metaregression. J Stroke Cerebrovasc Dis. 2020;29(8):104949.

60. Barrera FJ, Shekhar S, Wurth R, Moreno-Pena PJ, Ponce OJ, Hajdenberg M, et al. Prevalence of diabetes and hypertension and their associated risks for poor outcomes in COVID-19 patients. J Endocr Soc. 2020;4(9):bvaa102.

61. Zheng Z, Peng F, Xu B, Zhao J, Liu H, Peng J, et al. Risk factors of critical and mortal COVID-19 cases: a systematic literature review and meta-analysis. J Infect. 2020. 81(2):e16-25.

62. Baral R, White M, Vassiliou VS. Effect of renin-angiotensinaldosterone system inhibitors in patients with COVID-19: a systematic review and meta-analysis of 28,872 patients. Curr Atheroscler Rep. 2020;22(10):61. 